\title{
Téoros
}

Revue de recherche en tourisme

\section{Le tourisme dans les trois « capitales » du Brésil}

\section{Ana Maria Montenegro}

Volume 21, numéro 1, printemps 2002

Les villes capitales

URI : https://id.erudit.org/iderudit/1071535ar

DOI : https://doi.org/10.7202/1071535ar

Aller au sommaire du numéro

Éditeur(s)

Université du Québec à Montréal

ISSN

0712-8657 (imprimé)

1923-2705 (numérique)

Découvrir la revue

Citer cet article

Montenegro, A. M. (2002). Le tourisme dans les trois « capitales » du Brésil.

Téoros, 21(1), 21-28. https://doi.org/10.7202/1071535ar d'utilisation que vous pouvez consulter en ligne.

https://apropos.erudit.org/fr/usagers/politique-dutilisation/ 


\section{Le tourisme dans les trois " capitales " du Brésil"}

\section{Ana Maria Montenegro}

L e Brésil, pays-continent et république fédérative, a institué vingt-quatre capitales d'État, mais à l'échelle nationale, officiellement ou officieusement, trois capitales émergent : Brasilia, capitale fédérale, São Paulo, capitale économique, et Rio de Janeiro, restée capitale nationale sous bien des aspects. Ce sont ces trois villes qui font l'objet de cette étude. Sans doute ne doit-on pas oublier complètement les autres capitales d'État. Malgré les écarts régionaux de développement, de niveaux de vie et d'équipements, toutes ces villes rayonnent et attirent, ne serait-ce qu'à l'échelle de leur territoire, en raison de leur offre, en matière de commerce et de loisirs, et de leur appareil politique, administratif et culturel, voire financier. La Nouvelle Constitution de 1988 attribue aux gouvernements des États et des municipios des compétences et des prérogatives (Montenegro, 2000).

Si ces villes pâtissent de la distance/prix des voyages aériens, la situation en bord de mer est un atout considérable qui peut conduire au déploiement de l'aire d'attraction au-delà même des frontières nationales, à l'exemple de Florianópolis au Brésil austral et de Salvador de Bahia. Il en ressort aussi que les déplacements de loisir vers ces capitales relèvent du tourisme balnéaire, bien plus que du tourisme urbain, à l'exemple du Nordeste, où l'on part souvent en quête de la chaleur et du soleil d'hiver et également de la musique nordestine dont Caetano Veloso et Gilberto Gil sont les représentants les plus connus sur la scène internationale, Une nouvelle attirance désormais relayée par l'environnement. En conséquence, ces flux et les pratiques de loisir qui leur sont associées s'imposent comme un facteur important, voire décisif, des politiques urbaines.

\section{Un aperçu des marchés interne et externe}

D'après le nombre de billets vendus sur des vols intérieurs (22,5 millions en 1998; 22,2 millions en 1999; 24,2 millions en 2000), un Brésilien sur six prend l'avion une fois par année, comparativement à trois voyages par an et par habitant aux ÉtatsUnis $^{2}$. Voici quelques années, une association de consommateurs réclamait une baisse de $40 \%$ des prix et rếvélait qu'un aller-retour Săo Paulo-Londres acheté à l'étranger coûtait deux fois moins cher que le même billet acheté au Brésil auprès de la même compagnie. Sur les vols intérieurs, l'écart de prix pouvait atteindre $30 \%$ entre un billet acheté auprès de compagnies régionales et ce même billet vendu par les compagnies nationales ${ }^{3}$. Alors que le touriste étranger a pu se procurer les passesvoyages intérieurs chez lui, l'autocar s'impose au voyageur $\&$ national $*$ qui $n$ 'a pas accès au réseau de distribution ferroviạire. Selon l'entreprise brésilienne de tourisme Embratur, sur 157 millions de Brésiliens, 12 millions seulement font au moins un voyage annuel à l'intérieur du pays. Bien que ces flux intérieurs dépassent largement la demande touristique étrangère, celle-ci n'en est pas moins, la référence récurrente des politiques touristiques.

L'apport considérable du nord du continent (le Brésil est le quatrième pays visité par les touristes nord-américains et le septième par les des Canadiens ${ }^{4}$ ) ne l'emporte pas en volume et n'infirme pas les caractéristiques essentielles de la demande des pays frontaliers. Celle-ci relève surtout de l'interaction entre proximité géographique et culturelle ; par exemple, l'accès à des plages plus nombreuses et plus ensoleillées au Brésil qu'elles ne le sont au sud du continent. Florianópolis, capitale de l'État de Santa Catarina, est ainsi au troisième rang des villes les plus visitées par les touristes étrangers. Ces flux touristiques se prolongent sur Torres, Camboriu et d'autres villes balnéaires du sud et même jusqu'à Buzios, au nord-est de Rio, où Brigitte Bardot a naguère séjourné. . C'est surtout par la route que les Paraguayens, les Argentins et les Uruguayens accèdent au Brésil et gagnent la côte6. Cette clientèle forme, bon an mal an, presque la moitié des visiteurs étrangers. Bien que la domination du marché mitoyen sud-américain diminue à mesure que la distance s'accroit, Salvador, capitale de l'État de Bahia et quatrième ville en importance pour les arrivées internationales, est une des villes que les touristes Argentins visitent le plus souvent. Ce créneau que Recife, bénéficiant du voisinage d'Olinda, classée \& Patrimoine Mondial s, et Fortaleza, capitale du Ceara, voudraient bien exploiter même si elles misent aussi sur les brefs retours au pays de leurs émigrés respectifs, tout au moins ceux qui « ont réussi $"$.

Aujourd'hui «95 \% des touristes qui séjournent dans le Nordeste sont des Brésiliens; is viennent en autocar ou en voiture privée, ils logent chez des parents ou des amis; leur salaire mensuel moyen est de 2000 à $2300 B R L^{7}$. En juin 2001, 
à l'occasion de la Bumba-meu-boi, fẻte traditionnelle d'une dizaine de jours soutenue à gros renforts de marketing, 1'État du Maranhão, à la frontière du Nordeste et de l"Amazonie, recevait $20 \%$ de visiteurs de plus que l'année précédentés. D'autres villes organisent des carnavals hors saison... En fait, la concurrence s'accentue sur les côtes, dont les zones les plus proches et les plus facilement reliées aux capitales font, depuis une dizaine d'années, l'objet du programme a Prodetur-Nordeste: progranme de développement du tourisme * Celui-ci est doté d'un budget de $80 \mathrm{mil}$ lions de dollars américains, dont $50 \%$ sont empruntés à la Banque interaméricaine de développement, $24 \%$ en provenance de l'État fédéral par l'entremise d'Embratur et de la Banque Nationale du Développement Économique et Social. Le reste est financé par la Banco do Nordeste et le Trésor des États concernés (Becker, 1996). Ceci a sans doute contribué à la décision de I'Organisation Mondiale du Tourisme de réaliser sa réunion annuelle (en juin 2001) à Natal, capitale côtière du Rio Grande do Norte.

On ne peut pas en dire autant de Brasilia que bien peu de touristes étrangers visitent. En revanche, l'aéroport, fonction de capitale nationale oblige, est le troisiême du pays, desservi par une gare routière très fréquentée. . Pour sa part, Rio ne se départit pas du rôle de capitale du tourisme intérieur et extếrieur: l'occupation hôtelière y caracolait à $70 \%$ en 1998 , dix points de plus qu"en 1996, même en août, en a plein $\%$ hiver austral ${ }^{10}$. Ajoutons enfin que le « mouvement des aéroports de São Paulo, capitale de l'État du même nom, premier dans le pays, relève certes de son importance économique, mais aussi de sa position sur les routes aériennes vers le Centre-Ouest et l'Amazonie!".

\section{Tourisme dans la capitale fédérale... ville mystique!}

La hierarchie politique et administrative nous conduit d'abord à Brasiliá, capitale planifiée inaugurée dans les années Kubitschek, période clé du processus de développement du pays. Inscrite au patrimoine culturel de l'humanite, Brasilia ${ }^{12}$, dont le site a concentrerait $w$ des forces telluriques, est une ville empreinte de symboles, ce que les circuits touristiques mettent à profit, dans un esprit fort éclectique. Ces symboles conduisent de l'urbanisme et de l'architecture moderne de Lucio Costa et d'Oscar Niemeyer aux sources communes d'inspiration (I'eau comme composante de la forme urbaine, formes pyramidales dans l'architecture...) avec celles d'Akhetaton, capitale administrative de l'Égypte ancienne, dont la célérité de construction s'approcherait de Brasilia.

Troisième parc hôtelier du pays, avec 17000 lits, un taux d'occupation de l'ordre de $60 \%$, du lundi au jeudi seulement, rend bien compte d'un tourisme à dominante professionnelle lié surtout à la centralité du pouvoir (Cazes et Potier, 1996). Autant de données qui vont de pair d'une part avec la situation périphérique de Brasilia par rapport aux grandes villes et, d'autre part, les basses densités du Brésil central. Ces données expliquent la corrélation entre importance et nature du tourisme, développement du territoire, situation géographique face aux marchés ếmetteurs. L'hébergement et les autres services d"interêt touristique occupent ici $3,3 \%$ des actifs (29) 128 sur un total de 880000 , dont $91,5 \%$ dans le tertiaire), y compris les activités des villes-satellites. Composantes indissociables de l'économie de l'agglomération, leurs potentiels d'accueil et de services cadrent avec les moyens du plus grand nombre, résidants ou visiteurs réguliers.

Or, la renommée de Brasilia - et les agences de tourisme la présentent comme telle - est aussi celle d'une « capitale mystique *, voire a secrète $\%$, ce qui attire un grand nombre de touristes relativement démunis. Le temple de la Bonne Volonté, a pyamide à sept faces, au sommer couronné par le crislal de roche le plus pur jamais trowé au Brésill ( $18 \mathrm{~cm}$ de diametre, $40 \mathrm{~cm}$ de hautewr, 21 kilos)... $\%$, a longtemps été le monument le plus visité et conserve une honorable neuvième place. Peuton parler de concurrence vis-à-vis de la cathédrale métropolitaine (båtie par Oscar Niemeyer) et des nombreuses églises et chapelles catholiques de rite romain, comme l'ermitage dédié à Dom Bosco qui aurait, dès 1883, prévu l'avènement d'une a nouvelle civilisation entre le $20^{\circ}$ et le $15^{\circ}$ parallele $\mathrm{x}$ ? Une a quadra des religions ${ }^{13} \mathrm{w}$ concentre les sieges administratifs de diverses communautés religieuses à dominante syncrétique, alors que leurs centres de rassemblement et de vie trouvent leur place dans des lieux plus ou moins proches : Cidade di Palmelo (dans une des villessatellites où le spiritisme oriente la pratique de thérapies diverses), Cidade da Fraternidade (qui accueille et adopte des enfants), Cidade da Paz. Universidade Holistica Internacional de Brasilia à $26 \mathrm{~km}$ * qui ceuvre pour faire éveiller une nouvelle conscience et une nouvelle vision du monde $s$, Vale do Amanhecer a Vallée de l'Aube) à $42 \mathrm{~km}$ le plus grand phênomène syncrétique du pays, à certaines époques jusqu"à $3000-4000$ personnes s*y réunissent chaque jour... représentant des cultes et des entités afro-brésiliennes, égyptiennes, gitanes, incas, aztèques, mayas et même extraterrestres $\%$, Cidade Eclérica à $75 \mathrm{~km}$ liée à l'Union spiritualiste ligne blanche, * où toutes les religions s'unifient $*$ (Source : site Internet et guide officiel de la capitale).

Tour de ville "Brasilia secrète : 3 heures, déjeuner compris et guide bilingue (présenlé par l'agence Bhepoint)

Venez connaitre les beauls el l'aura mystique de la Capitale federale et ses merveilles architecturales. Plas de 700 institurions ésotériques, religieuses et philosophiques ont migre vers le Plateau Ceniral brésilien et y forment whe babel de courants qui atrire chaque fois davantage l'attention des touristes et des * amants de spiritualite. ${ }^{14}$

Si la notoriété du religieux comme composante de l'attractivité de la ville renvoie au tourisme de congrès et de rúunions, cette préminence contraste avec le profil-type des visiteurs de Brasilia, dressé dans une enquête de 2001 , ou plus exactement de ceux logeant à l'hötel (ils ne sont que $48 \%$ ). Ces voyageurs ont majoritairement leurs 
frais payés et ont choisis un hôtel d'au moins trois étoiles. Ceux-ci sont a $65 \%$ de sexe masculin et mariés, $50 \%$ ont entre 30 et 40 ans, et détiennent un diplôme universitaire ou l'equivalent. Ils sont chef d'entreprise ou ont une profession libérale. Ils voyagent en avion $(57 \%)$, le plus souvent seul $(56 \%)$, achètent leur billet directement d'une compagnie aérienne et le réccupèrent à l'aéroport, sans avoir recours à une agence de voyage. Ils vivent souvent dans la région Sudeste, se rendent à Brasilia pour travail/affaires/congres, leur revenu moyen mensuel est de $3001 \mathrm{BRL}^{15}$. Par ailleurs, toujours selon la même enquête reprise depuis cinq ans, soulignons qu'il y a $7,5 \%$ d'etudiants parmi ces voyageurs. L'université de Brasilia, qui préserve toujours le souvenir de la répression militaire, $s$ 'est acquis une renommée considerable et contribue à son tour à la multiplication de congrès et réunions universitaires ou autres, $s^{4} y$ multiplient. L'activite du seul Convention \& Visitors Bureau en témoigne puisqu'il a attiré 6 événements en 1998, contre 36 et 40 les deux annés suivantes, réunissant respectivement 3600,39900 et 54000 participants, sans oublier le congrès annuel de l'Agence brésilienne des agents de voyage en 2001 . La ville ne manque certes pas de centres de congrès ni de musés. Cependant, l'apport des touristes étrangers a

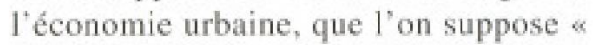
passage obligé $x$ en matière de relations diplomatiques, voire économiques, est « insignifiant $*$ : peu nombreux à visiter Brasiliạ $(4,5 \%$ à la dernière enquête $)$, ils n'y font souvent qu' un tour de ville, le temps d'une escale avant de reprendre leur vol vers d'autres contrées (Paviani, 1995).

\section{Les villes-capitales \\ les plus peuplées du pays: entre tourisme d'affaires et de loisir}

\section{São Paulo}

Capitale économique du pays et première agglomération d'Amérique du Sud, São Paulo est devenue depuis six ou sept ans la grande porte d'entrée du pays et le lieu de départ de plus de $40 \%$ des touristes brésiliens ver's l'étranger ${ }^{16}$, Beaucoup de débarquements dans ses aéroports relèvent toutefois de transferts sur des vols intérieurs. Par ailleurs, l'attraction de la ville est mitigée pour ceux qui arrivent par la route de la zone de la triple frontière avec l'Argentine et le Paraguay, en direction surtout de Foz do Iguaçu. ${ }^{17}$...

São Paulo, qui cumule les palmares en matière d'économie et de croissance, s'evertue à retenir ses « visiteurs en veslon et cravate w au-dela du temps necessaire aux affaires... Lintéressé a ne songe pas à rester ne serait-ce que le temps d'un week-end w. Aussi voit-on se multiplier les incitatifs à prolonger les séjours : Visa a parfois proposé des remises (jusqu'à $50 \%$ ) surt le prix du voyage et de l'hébergement et des a cadeaux $\%$ dans 120 établissements commerciaux (restaurants, hôtels, bars, bars-tabac, librairies, location de voiture). Trois millions de dollars américains ont été alloues à ce programme d'une année, au besoin davantage, auquel se sont associes le Convention \& Vistors Bureau, Varig et Embratur, dans l'objectif a d'inciter les entrepreneurs et les hommes d'affaires a rester dans la ville apres avoir participé à des réunions et des événements qui les $y$ ont amenés $\$$ (OESP, 18 avril 1997). Par la suite on annonçait l'arrivée, au cours des cinq années suivantes, de cinq entreprises d'organisation de grandes foires car la ville est le plus grand centre d'événements d'Amérique latine, promoteur d'an tourisme qui a brasse 3 milliards de dollars américains en 1998 d'après la revue US Update de la Chambre anéricaine * (FSP, 2 aout 1999). * En matiere d'activités parement commerciales autant que sous sa modalité sociale, le potentiel de la ville est indiscutable $\%$, affirme-t-on! Même si leur revenu moyen annuel dépasse celui des autres voyageurs, il n'en reste pas moins hasardeux d'affirmer que $\propto 90 \%$ de ceux qui y debarquent viennent pow offaires et peavent se permettre de depenser 240 \$US/jour, trois fois plus que le visiteu traditionel (FSP, 1 juin 1997)18, car seulement $52 \%$ des arrives internationales relevent des affaires. Près de 45000 foires, congrès, événements ont lieu annuellement dans l'État de Siro Paulo dont la majorité dans là capitale où ces activitếs suscitent $60 \%$ de l'occupation hôtelière. Il y aurait de quoi attirer des capitaux japonais qui pourraient ainsi investir dans des complexes touristiques de l'Etat. comme au Nicaragua... Les échanges avec le Texas, Buenos Aires, le Japon devraient s'accroître, confirmait le Secrétaire aux Relations internationales de l'actuel gouvernement municipa $1^{19}$. Rappelons à ce propos que trois musées de la ville sont dédiés à l'immigration - du Japon, de l'Italie et de tous les autres pays dont les ressortissants sont venus pour travailler la terre, sur l initiative du gouvernement de l'État, vers la fin du XIX - et que a l'hôtel w où on les conduisait à leur arrivée abrite aujourd'hui le Mémorial de l' Immigrant ${ }^{20}$.

Le touriste se perd dans whe ville ou l'information à son intention est insuffsante et où personne ou presque ne parle l'anglas $\%$. Devant ce constat, l'entreprise municipale Anhembi Turismo avait prévu la mise en place de plaques d' informationorientation, de cours destinés aux conducteurs de taxis, aux policiers et aux étudiants, de campagne télévisuelle sur l'importance du tourisme... : cette entreprise s'etait donne pour objectif d'asseoir l'image de la ville comme capitale de la gastronomie ${ }^{21}$, undes plus grands centres gasironomiques du monde , selon une publicité récente signée Embratur. De ce point de vue, l'apport migratoire est encore un elément important de la culture urbaine, malgré la montée, au cour's des dernières années, de discrimination contre les nordestinos (Pires, 2001). D'ailleurs, les flux touristiques en provenance du Nordeste se renforcent, tout au moins à l'occasion d'événements porteurs comme la course automobile à Interlagos (rapatriée de Rio depuis une dizaine d'années) ou lorsque des hommes d'affaires accompagnés de leurs femmes y viennent pour, entre autres, faire leurs achats...

São Paulo, tremplin latin.., chaque jour 300 clientes venues des quatre coins de la planète dépensent jus$q u ' a$ 1,5 milion de francs 1250000 euro) chez Dasla, La boutique la plus laxueuse du Bresil... la Morumbi Fashion Week peaufine sa renommé... Les créareurs paulistes conquiènent Les podiwns etrangers ! $\times$ São Paulo, le tremplin Latino s, Le Monde, 10 mars 2001). 
Andrade et Rodrigues, pour leur part, ont portế leur regard sur les sources d'une image qu'ils considèrent réductrice : « la ville des affaires $\%$. Cette étiquette est à metrre sur le comple de cerfains intérêts... qui misent sur les grands centres commerciaux, au détriment des options culturelles, historiques et de loisir .. Purement $*$ technocratique \%, elle déteint sur lä ville et conduit les habitants à l'assimiler tout entière à la seule avenue Paulista, bordée par le siège des banques, les hôtels de luxe, les restaurants et les boutiques... A moins d'admettre que a la dynamique de la ville entraine et galvanise aussi les activités touristiques $x$ (Andrade et Rodrigues, $1995: 3,7)$ ? Cette hypothese semble être confirmée par les expositions présentées jusqu'en janvier (Cazes, 1996 :62-65).

Au 1578 de la Paulista, l'épine dorsale de la ville, le Musée d'Art, le MASP, I'un des plus importanis musées de l'Amérique du Sud, affiche hau sur ses pilotis une exposition d'an egyptien choisi dans les collections du Louvre... Loin de là, la Pinacolheque, un modele récent de muséographie, expose Rodin ; enfin, dans le Panc Ibirapuera, l'Oca, don l'architecture de science-fiction est signée Oscar Niemeyer, accueille a Parade whe exposition issue des collections du Centre Pompidou, grâce au banquier et mécène Edemar Cid Ferreira, figure controversé de l'art et du patrimoine brésiliens ( Quand la France parade à São Paulo *. Le Monde, 3 novembre 2001).

Capitale économique et culturelle du Bresil - 620 millions de franos 194,5 millions euro) sont collectés par des fonds privés pour financer la création artistique, São Paulo se remet peu à peu des années de dictature et des crises à répétinion... (* São Paulo, le tremplin latino, Le Monde, 10 mars 2001).

Il est aussi vrai que * la * Paulista, qui a détrôné l'ancien centre, doit à son tour tenir compte d'un nouveau centre d'affaires, le Berrini, qui s"impose au sud-ouest sur des terrains où le prix du mètre carré est digne d'une capitale des affaires... sur la Marginale qui longe le Pinheiros, cette * rivière désespérément polluée qui traverse la ville (Rochefort, 2000: 138) avec des voies qui la longent, souvent encombrées:

São Paulo vit dans un rythme suicidaire... la cinculation y est infernale, des fous foncent dans toutes les directions... résumons-nous, $n$ 'y restez pas plus de 48 heures... Mieux vaut éviter São Paulo, j'y ai élé el ma seule envie était de m'enfuir22 !

Ces commentaires, glanés dans divers guides touristiques étrangers (toujours d'après la même analyse), montrent à quel point penser le tourisme en fonction du seul touriste étranger, dans une métropole comme São Paulo, est une erreur courante car l'habitant est, lui aussi, un grand consommateur d'activités culturelles dites touristiques. Le centre d'information touristique de la place de la République en tếmoigne : il rêpond chaque jour à environ une trentaine de touristes étrangers, à 20 brésiliens non-paulistes et à 110 habi= tants de la ville ! De quoi admettre, à l'instar d'Andrade et Rodrigues $(1995: 6,1)^{23}$, que l'image touristique peut rendre compte des désirs que suscite cette capitale où tout semble possible, où tout peut arriver... comme a devenir quelqu'un $*$ ou, tout simplement, avoir du travail, alors même que cette ville $\alpha$ n'a pas de mémoire $\%$.

\section{Rio de Janeiro}

Ce n'est pas le cas de Rio, riche de la mémoire d'une capitale fédérale ! Par le passe si attractive, Rio s'est transformé à un point tel qu'on l'a même déjầ accusée, dans la seconde moitiế des années 1980 , de la a fuitè générale des touristes, étrangers surtout, qui craignaient pour leur sécuritêe ${ }^{24}$. En Argentine, par ailleurs, * pays froid aux zones balnéaires restreintes comparées à celles du Brésil, le marché touristique avait le vent en poupe! $\mathrm{s}$ (OESP, 26 décembre 2000). Le ton était donné, malgré les interprétations différentes : pour Embratur, le problème venait de 1 'insuffisance de publicité et d'informa- tion à l'étranger. Conjuntura Econòmica, revue de la Fundaçao Getulio Vargas, institution de recherche renommé, accusait : * en 1990 Rio recevait $51 \%$ des touristes etrangers, contre seulement 39 \% en 1994... w (Teixeira, $1994: 59-61)$. Cette redistribution partielle sur d'autres lieux et régions du pays est souhaitable et sou= haitée, tout au moins à en juger par les politiques en lạ matière, qu'elles soient le fait de la Nation, des Etats ou des municipalités.

L'image négative de Rio se transpose au pays : en tant que destination touristique le Brésil est o hors course (OESP, 7 novembre 1995).

En deur ans, de 1988 à 1990, le Bressil a perdu 651000 visiteurs etrangers, whe chute de $37 \%$; pendant cente même période, le nombre d'assassinals a doublé à Rio... de 1987 à 1994 les arrives au Brésil ont diminué de 17 \%et les homicides à Rio ont plus que triple... (Veja, 6 décembre 1995).

Le pays atrire moins que l' Uruguay. ...que la Jamaíque, une très perite île des Caraibes.. qui alloue 30 millions $\$ U S$ à la promotion sur les marchés extérieurs ; le Brésil devrait suive cet exemple. La violence sur les plages de Rio effraie les touristes nord-américains dont les arrivées ont chute a 60000 , contre 180000 en 1960... La Fondation de l'industrie touristique (privée) salue les Etats du Nordeste qui se sont batrus pour insérer Natal, Fortaleza, Maceio sur la carle touristique mondiale; ces villes pourratent concurrencer Rio comme porte d'entrée du pays (FSP, 18 février 1996).

Un doute persiste en ce qui concerne l'Uruguay, étant donné la taille réciproque des deux pays, alors que pour la Jamaïque, paradis fiscal livré aux touristes $\mathrm{d}^{+}$k affaires et aux autres vacanciers americains, la comparaison est presque infamante pour le Brésil ! En effet, selon Le Monde du 10 mars 2001, Rio retrouve de son importance 
mêne si les recettes du tourisme en 1994, atteignent $21 \%$ du PIB de I'Etat de Rio de Janeiro (50 milliards \$US cette annele) contre $28 \%$ en 1984..., on peut s'attendre dे un processus de réversion de l'image négative de cette capitale, symbole de la violence urbaine mondiale, comme New York certes, si ce n'est que les recherches sur Rio temoignent tout de même de l'efficacité des forces policieres dans la ville.

Par ailleurs, \& [...] le taux d'occupation hôteliere s'y redresse: 51,6 \% en 1994 , $62 \%$ et $63 \%$ les années d'après, $70 \%$ en 1997... * (Carta do Rio, janvier 1998). En effet, le plan Rio Maravilha, élaboré dès 1997 par la municipalité et les entreprises du secteur touristique, décidait d'investir 15 millions de dollars américains en publicite et se donnait comme objectif, à l'horizon 2000, d'attirer 5 millions de brésiliens et 2 millions de touristes étrangers en provenance de villes ciblées dans différents pays. Ces derniers sont venus, au nombre de 734000 en 1998, puis de 807 000 l'annêe suivante, comparativement à 4,2 millions de Brésiliens, au moment ou la ville se plaçait septième au classement de I'International Conferences and Conventions Association, en accueillant 60 événements internationaux 25. Déjà le Rio Visitors and Convetion Bureau annonçait plus de 80 contrats signés pour l'annéce 2000 et 300 autres prévus jusqu'en 2007. D'après Rio Maravilha $a^{36}$ qui revendique « une année de discussion avec la population $x$ et se veut

le plan du carioca et non pas celui du gouvernement, les aspects de la dynamique urbaine à prendre en compte en matière de facteur négatif pour le tourisme seraient : (1) l'importance des bidonvilles et de la criminalité qui l'accompagne (sic); (2) l'emprise du transport par autobus qui transforme certains quartiers en couloirs de passage; (3) l'eloignement de la Zone Ouest à cause de l'expansion désordonnée de la ville.

Or, ce dernier point pose problème car, du moins en partie, cette expansion relève des plans d'urbanisme qui la reconnaissent comme seule réserve d'espace et seule aire de desserrement démographique dans lä ville. Un diagnostic des problemes et insuffisances conclut avec une affirmation discutable : le ciroyen carioca $n^{3}$ a pas conscience de l'importance du tourisme pour la ville w. Or, depuis fort longtemps, qu'il soit étranger ou brésilien, le touriste s'observe partout dans la ville, souvent pour des séjours réguliers auprès de sa famille. Cela aussi fait partie du savoirvivre et de la mémoire du carioca (Montenegro, 2001).

Quoi qu'il en soit, le produit touristique défini pour Rio s'appuie sur des éléments de base (beauté naturelle, équipements, urbanité, histoire urbaine, modernité, attirance en matière d' 6 vénements internationaux et nationaux...) conjugués en fonction des publics cibles et de la durée des séjours. Des produits, territoriaux ou thématiques sont censés correspondre au a concept * Rio Maravilha, y compris auprès du carioca, invité à a mieux goûter sa ville $\%$ (comme c'est aussi le cas pour le paulistano, c'est-àdire le citoyen de la ville de São Paulo évoqué ci-dessus).

La mise en ouvre d'un tel plan comporte de nouvelles installations (aquarium, parcs à thème...), alors que d'autres sont à aménager, comme le Fort de Sao Joao, sur le cap qui délimite entre elles les plages de Copacabana et d'Ipanema, déjà ouvert au public. Le fort s'intégrerait à un futur parc thématique centré sur la baie de Guanabara, avec d'autres élé́ments du patrimoine. Le site Internet du Secrétariat municipal au Tourisme signale déjà la progression des visites et des promenades en bateau, à proximité de la façade maritime du centre-ville. Les services comportent, notamment : l'autobus touristique qui dessert des points d'intérêt plus ou moins éloignés des zones centrales, ou d'accès difficile sans voiture ; l'accès et $1^{+}$equipement rénovés du Pain de sucre (484 442 entrées, avant mếme les grandes vacances de fin de l'année 2001) ; le programme " Connaître Rio à pied $*$ (qui concerne surtout le centre-ville, dont le riche patrimoine urbanistique, architectural et muséographique a bênéficié d'une rénovation de qualité : création ou renouveau des musées; les réductions d'impôts que préconisait Celso Furatdo, alors ministre de la Culture, y ont \& fait merveille w) (Montenegro, 1995). Le vieux centre a enfin $\alpha$ recrée ses ressources et offre... une recréation de qualité aux populations locales $\%$. C"est dire que par son cadre patrimonial aussi, architecture moderne comprise, malgré sa taille et son histoire rếcente, Rio a bien des caractéristiques des a villes d'arts $\%$ et des « petites villes écrin * que décrivent Michel Dewailly et Émile Flamant (2000: 116, 121). Rio est bien placée en matière de tourisme écologique, malgré la catastrophe pétrolière dans la baie en janvier 2000) face à d'autres grandes villes du pays. En revanche, la violence s'est immiscée dans l'image qu'on en projette...

Enfin, en ce qui concerne le Plan Maravilha, il ne faut pas oublier, parmi ses composantes (information, marketing), celles qui touchent, alternativement ou de manière plus spécifique, les touristes et les professionnels, comme la mise en place ou la rénovation d'hốtels ${ }^{27}$ mềme si l'on peut craindre qu'elle ne témoigne de la «précipitation déraisonnable \$ qu"évoque Georges Cazes (1996: 54$)$ à propos de certaines villes moyennes européennes, où « le phénomène de surcapacité peut déboucher sur une veritable saturation du marché $\%$ qui est inséparable de la nécessité pour les chaînes hôtelières de s'affirmer partout. A propos des partis pris d'une planification stratégique omniprésente, le quotidien O Globo (19 décembre 1998) s'exclamait : « les groupes hôteliers ont de quoi se réjouir! $w$.

Quant à Rio, rappelons qu'au moment du Réveillon 2002, qui coïncidait avec la commémoration des 500 ans de la Baie, 6 millions de reales brésiliens (l'équivalent de 2556782 \$US), auront été attribués à son financement dont les troisquarts de cette somme pour Copacabana, où l'on attendait 3,5 millions de personnes, dont $20 \%$ d'étrangers... A moins que, en raison de la tragédie du 11 septembre, ces prévisions n'aient été revues à la baisse... 


\section{CONCLUSION}

La comparaison proposée par Téoros sur la réalité touristique des capitales nationales et celle des autres centres touristiques d'un pays nous a conduits de la capitale fédérale, Brasilia, à Rio, l'ex-capitale à l'attractivité sans cesse confirmée, où la rénovation $\mathrm{du}$ a vieux centre $\%$ suggère peut-être une comparaison avec le VieuxQuébec et, enfin, à nous intéresser à Sāo Paulo. Aux commandes de l'économie nationale - Milton Santos l'a montré et théorisé en géographe (Santos, $1994 \mathrm{et}$ 1992) -, São Paulo se présente en capitale du tourisme d'affaires. Mais comment en discuter à partir dı seul décompte des arrivées déclarées pour affaires comme « motif principal du séjout $\%$. Bien qué ce critère soit entaché d'insuffisance et d'imprécision, il est pourtant mis généralement de l'avant pour justifier la rentabilite de certains services touristiques, en particulier dans l'hotellerie et la restauration what de gamme *. En conséquence, São Paulo ne se range-t-elle pas parmi « ces villes persuadées, a tort ou à raison, de patir d'une manvaise image de marque, (où) la volonté de reconsidérer la représentation collective de l'agglomération et, au besoin. de la reconstruire tolalement avant d'en faire un instrument de promotion, est une des justifications le plus souvent avancées pour investir dans le développement tou= ristique * (Cazes, $1996: 47-48)$ ?

La réflexion de Menezes (1996: 98) à propos du label patrimoine de l'humanite "lorsqu'il ne fonctionne pas comme tel à l'échelle du local, du municipal, du régional $\%$, renvoie à Brasilia: quel en est le sens, selon qu'on y a son lieu de vie et de travail, ou qu'on ne s'y rende que pour accomplir des tấches le plus souvent humbles et mal payées? L'intéressé « ne songe pas à rester ne serait-ce que le temps d' ${ }^{\prime}$ ' week-end *. Car le fossé est en effet immense, entre la capitale nationale - où les niveaux de vie. d'instruction et de salaires l'emportent sur le pays tout entier et les villes-satellites et autres, à la fois distantes et dépendantes de son offre de travail. On le sait, dès l'annonce de la construction de la future capitale du pays, des flux nourris et constants, formés par des travailleurs en provenance d'un peu partout, se sont dirigés et relayés vers ce plateau lointain et faiblement habité. Ces émigrants se trouvaient souvent déracinés. tout au moins jusqu'au moment d'y faire venir leur famille ou d'en créer une sur place. Ce déracinement n'est-il pas un facteur explicatif majeur de l'importance acquise ici par le religieux qui est de toute façon, à travers la diversité de ses formes d'expression, une donne fondamentale de la culture et du quotidien de la majorité des Brésiliens ?

Finalement, à propos de Rio, rappelons les lignes de rupture, en termes de distance, d'urbanisme et de modes de vie, mais aussi en termes de génération, entre les quartiers traditionnels de Rio, aisés ou non, plus ou moins centraux et les condominiums fermés x (Montenegro, 2000 et 1993), centres commerciaux, parcs de loisir et autres microcosmes, y compris universitaires, de Barra da Tijuca où de nouvelles et assez nombreuses générations de cariocas sont nées ou ont grandi. Notons enfin, la rupture sociale et économique entre « la Barra $*$ et les zones populaires environnantes et plus ou moins proches qui lui foumissent largement sa main-d'cuvre. On sait par ailleurs que bien des investissements y sont faits en vue des vacances et des week-ends, tant pour des voisins sudaméricains, que pour des cariocas. Tout cela fait se demander si ces formes de fragmentation, perceptibles depuis longtemps, auront leur version touristique, à moins qu'elles ne l'aient déjà. À suivre..

Ana Maria Montenegro est maitre de conférence au Département de géographie, Université Paris XII, et résorière de la Commission de géographie du tourisme et des loisirs au Comité nainonal français de gégraphie.

\section{Notes}

1 L'ensemble des citations provenant de textes en portugais sont des traductions libres de l'auteur du présent article.

$2 J B, 12$ août 2001

$3 J B, 27$ juillet 1996.

4 En 1998,527 000 arrivées en provenance des États-Unis, une hausse de 31 \% sur I'année précédente, el 47000 arrivées du Canada, une hausse de $60,2 \%(219,89$ \$US/jour) ; ces touristes sont aussi parmi ceux qui dépensent le plus. Leur apport atteint cette année \|I \% et $2.5 \%$ respectivement du total des arrivếes étrangères (OMT, 1999).

5 En 1999, 8,5 \% des touristes argentins au Brésil et $6 \%$ des Français ont visité ce site balnéaire snob situé à $150 \mathrm{~km}$ au nord-est de Rio.

6 A ce propos, lire Montenegro (1994). En 1999, arrivés au Brésil en provenance des pays de La Plata (Argentine, Paraguay, Uruguay) : par voie terrestre 1750204 arrivées sur 2,446000 (soit $71,5 \%$ ) ; en ajoutant la Bolivie, on obtient 1874492 ; par avion 632000 (soit $25 \%$ ) sur 2534000 .

Source : Embratur, Anuario Estatisfico (2000) (idem pour toutes les arrivées. intermationales et intérieures, quel que soit le mode de transport).

7 Enquête Sudene, d'après le quotidien Gazeta Mercantil, 25 septembre 1997.

8 Selon l'hebdomadaire Epoca, 2 juillet 2001 .

9 Brasilia : (1) vols internationaux, au débarquement : 7804 passagers en 1998, 1 384 en 1999 , soit $2.50 \%$ et $1.78 \%$ des touristes étrangers au Brésil ont visité la ville, empruntant majoritairement des wols in. térieurs. Sur 162 vols/jour sur Brasilią, deus seulement assurent des liaisons internationales, avec Miami et Buenos Aires ; (2) vols intếrieurs, au débarquement

Brasilia : 1902831 passagers en 1998. 1927266 en $1999: 2394000$ en 2000 .

Rio : 2884801 et 3429320 passagers: Săo Paulo : 5124928 et 6253560 passagers.

10 O Globo, 19 décembre 1999.

11 Aéroports de Săo Paulo - vols intérieurs. débarquements: Congonhas : 2 114,6(1998), 
3078,7 (1999): São Paulo: 3010,3 (1998), $3174,8(1999)$.

12 Sources des donnees sur Brasilia ; site Internet du Brasilia Convention \& Visitors Bureau : Perfil do turista do Distrito Federal : Brasilia Tourist Guide 2001 ; Kern et Pimentel.

13 Quadra : ensemble de pâtés de maison * équipés w, earacteristiques de Brasilia.

14 Conheça todas as belezas e a aura mística da Capital Federal, com seus encantos a maravilhas arquitetônicas. Mais de 700 organizaçōes esotéricas, religiosas o filosóficas migram para o Planalto Central brasileiro, formando uma babel de correntes que chama cada vez mais a atençĩo dos turistas c amantes da espiritualidade.

15 La demiere enquête date du premier trimestre 2001 ; en octobre, le dollar oscillait de 2,66 a $2,85 \mathrm{BRL}$

16 Depuis une vingtaine d'années, la majorité des vols en provenance de l'ctranger se posent d'abord à São Paulo, et non plus â Rio, fin de parcours pour une partie seulement des vols.

17 São Paulo n'attire pas, ou pas assez, ceux qui arrivent aux frontières Argentine-Paraguay-Brésil par la route, en direction de Fozdo Iguaçu, ville où la mise en place de la centrale hydroélectrique d"Itaipu (considérée la plus grande du monde) a provoqué une croissance exponentielle. Ses 275 chutes de 40-80 mètres de haut, classées dans le patrimoine mondial de l'humanité, en font une valeur sûre des circuits touristiques guidés. Cinquiême parmi les villes les plus visitées en 1998 et 1999 par les touristes étrangers, Foz héberge aussi les Brésiliens qui se rendent à Ciudad del Este, au Paraguay, pour s'y procurer des produits importếs d'origines diverses qu'ils ramènent plus ou moins lếgalement au Brésil. Cependant, les routes qui relient cette zone au littoral par l'État du Paraná et celui de Sāo Paulo ne favorisent pas l'accès à sa capitale que bien de bretelles permettent de contourner (Salomon, 1992).

18 Les chiffres correspondants seraient de 66 500 dollars américains pour 537841 personnes (soit $26 \%$ du total des entrés) contre, à l'échelle du Brésil, 41500 \$US en 1996 et 39600 \$US en 1995. Motifs des voyages a Salo Paulo: étrangers arrivés sur des vols internationaux : affaires $51,9 \%$, tourisme $37,6 \%$, evenements $7,8 \%$, autres $2,7 \%$; arrives sur des vols intérieurs et aux gares routières (75\% de passagers brésiliens) : visite de parents et d'amis $31 \%$, affaires $21 \%$, loisirs el achats $21 \%$, evénements $5 \%$, traitements de sante $7 \%$, autres $15 \%$ Source: Secretaria de Esportes e Turismo do Estado de Sáo Paulo.

19 Serson, J. (2000) : 232-239 ; FSP, 2 janvier 2001. A noter, en 1997-1998, une baisse des arrives en provenance du Japon (-41 \%), réduites à 23100 en 1998 , ainsi que du Canada $(-14,1$ \%) et des EtatsUnis $(-9,0 \%)$, avee respectivenent 485700 ct 4885500 entrées en 1998 (OMT, 1999).

20 FSP, I juin 1997 FSP, 25 décembre 1997.

21 A titre d'exemple el sans commentaires... a Après l'immigration européenne est venue celle du Nordeste... Le progres économique a apporté à Sáo Paulo à la fois du progrès et de la misère, la rationalisation de l'espace et le chaos urbain... Comment le principal centre développé du pays est-il devenu un autre pays, sans douanes et sans restrictions ả l'entrée ? São Paulo est devenue la plus grande ville nordestine du pays et a fait ainsi proliférer la misère et une détérioration scandaleuse du niveau de vie... Un seul Etat, encore moins une ville, est incapable d'absorber toute la main-d'ceuvre du pays o (Pires, Lazer e turismo cultural, a Préserver le patrimoine culturel de São Paulo: difficultés de la richesse $\mathrm{n}, \mathrm{p} .103$ ).

22 Andrade, J., et Rodrigues, C.N., p. 5-6, d'après Le Guide du Routard Bresil, Hachette et Club Guide, Italie, cités par FSP 8 septembre 1994, p. 3-1.

$23 *$ Ceux qui vivent à Săo Paulo n'y sont pas pour souffrir à faire la queue, affronter le transit, la pollution, l'insuffisance en matière de logement, la violence. Non, le tableau du bonheur est assez bien encadre Il suffit de regarder. II suffit de désirer pour essayer. Et ils croient tous que la ville leur en donnera I'occasion $s$, (Andrade et Rodrigues, 1995-6, d'après Lima, 1990).

24 OESP, 26 décembre 2000

25 Embratur, Anuario Estatistico 2000. dossier et site Intemet du Rio Conven- tion \& Visitors Bureau, site Internet du Secretaria de Turismo - Riotur.

Ville de Rio de Janeiro:

Equipenents et services

Hôtels: 22000 chambres dams 188 hôtels classés de 1 à 5 étoiles.

Taux d'occupation hôtelière : 1994 $57,6 \%, 1995-65 \%$, ces dernières années $-68 \%$; 277 auditorium-salles de réunions Equipes (26 922 places).

Centres de congrès et d'expositions : Riocentro, $100209 \mathrm{~m} 2$, le plus grand centre des congres de l'Amérique latine

Selon la municipalité, il manque à la ville un centre de congrès de capacité moyenne: un projet serait en voie d'approbation, à proximité du CBD, à côté du Téléport (liré, sur ce quartier, Montenegro, 2000).

Agences de voyage : 350 , dont 60 spécialistes du réceptif.

Points d'information touristique $(2000)$ : accueil de 37800 personnes.

4. ALO RIO 1035 * ; renseignements têléphoniques; prospectus envoyés sur demande : 403713.

26 Par sa conception, ses méthodes et les acteurs auxquels il fait appel - dont la participation est censée fonder sa légitimitế - la démarche du Plano Maravilha (voir Bibliographie) reproduit celle mise en eeuvre par le Plan stratégique de Rio de Janeito (Montenegro, 2000).

\section{Bibliographie}

Andrade, Julia, et Cintia Nigro Rodrigues (1995), * Por uma reapropriaça da cidade: especulaça sôbre a imagem da cidade de São Paulo trabalhada pela industria do turismo $*$ Congresso international de Geografia e planejamento do urismo; Sol e territorio. Département de Géographie, Universidade de São Paulo-USP, 16-22 juillet, 12 p. polycopiées.

Becker, Bertha (1996), \& Politicas e planejamento do turismo no Brasil \%, dans Eduardo Yazigi. Ana Fani Alessandri Carlos, Rita de Cassia A. Da Cruz (dir), Turismo, wma pratica entre a crise a a hovacao na metropole do Rio de Joneiro, Turismo, espaço, paisagem e cultura, Sao Paulo, Hucitec, p. 181-192.

Cazes, Georges, et Françoise Potier (1996), Le 
tourisme urbain, Paris: Presses Universitaires de France, coll. Que sais-je?

De Menezes, Ulpiano T. Bezerra (1996), \& Os "usos culturais" da cultura. Contribuiçao para uma abordagem critica das praticas e politicas culturais $\%$ dans Eduardo Yazigi, Ana Fani Alessandri Carlos et Rita de Cassia A. Da Cruz (dir.), Turismo, wma pratica entre a crise e a inovaçao na metropole do Rio de Janeiro, Turismo, espaço, paisagem e cultura, São Paulo, Hucitec, p. 88-99.

Dewailly, Jean-Michel, et Emile Flament (2000), Le Tourisme, Paris, coll. Campus, Sedes.

Lima, J.C. (1990), Fragmentos de um discurso urbano, Dossic Cidades. Universidade de Săo Paulo-USP

Montenegro, Ana Maria (2001), o Identité urbaine et pratiques festives à Rio de Janeiro *, Paris, Geographie et Cultures, n 39 , p. 53-66.

Montenegro, Ana Maria (1995), “ Evoluçao recente da demanda turistica internacional e mercado para o Brasil, São Paulo w, Congresso international de Geografía e planejamento do turismo: Sol e ferritorio, Département de Géographie, Universidade de São Paulo-USP, 16 22 juillet, p. 20 polycopiées, tableaux.

Montenegro, Ana Maria (1995), « Servici socioculturali e pratiche del tempo libero à Rio de Janeiro : nuovi spazi, nuovi segregazione *, dans R. Cathedra et M. Memoli (dir.), La Citta ineguale. Pratiche culturali e organizzazione della marginalita in Africa e America Latina, Milano: Ed. Unicopli, p. 139-154.

Montenegro, Ana Maria (1994), * Pour une approche du tourisme intérieur brésilien s, Le tourisme non-littoral dans les pays en développement, Colloque de Marrakech 20-24 octobre 1991, Revue de la Faculté de Letires et des Sciences Humaines, $\mathrm{n}^{\circ}$ 10, p. 219-236, cartes.

Montenegro, Ana Maria (1994), * Le Tourisme international au Brésil *. Paris : L'Information Gégraphique, vol. 58, $n^{\circ}$ 5. p. 199-210.

Montenegro, Ana Maria (1993), * Tourisme, loisirs et urbanisation sur le littoral de Rio de Janeiro $*$, Recherches Urbaines, $\mathrm{n}^{\circ} 8, *$ Architecture, urbanisme et amenagement en mi lieu touristique littoral s, Cesurb, Talence, p. 103118 , cartes.

Montenegro, Ana Maria, et Dominique Rivière (2001), * Naples et Rio : "renouveler la ville" quels enjeux pour l' identité urbaine ? " dans
Renouveler la ville: les enjeux de la régénération urbaine, Fréville publications, Pôle Universitaire Européen Lille-Nord-Pas de Calais, p. 272-286.

Paviani, Aldo (1995), \& Turismo nas métropoles: potencialidades novas? , Congresso international de Geografia e planejamento do turismo: Sol e territorio, Departement de Géographie, Universidade de São Paulo-USP, 16-22 juillet. 5 p. polycopićes.

Pires, Mario Jorge (2001), Lazer et turismo cultural, Barweri (SP), Editora Manole Ltda, $130 \mathrm{p}$.

Pires, Mario Jorge (2001), Preservar o patrimonio cultural de São Paulo: a dificul= dade da riqueza, p. $101-105$.

Plano Maravilha, Plano de turismo da cidade do Rio de Janeiro, Relatorio de progresso, aoutt 1999, Prefeitura da Cidade do Rio de Janeiro, $45 \mathrm{p}$.

Plano Maravilha, Plano de turismo da cidade do Rio de Janeiro, Relatorio final, décembre 1997. Prefeitura da Cidade do Rio de Janeiro, $182 \mathrm{p}$.

Ribeiro, Ana Clara T, D Catia A. T. da Silva, Hermani de Moraes Vieira, et Rita de C. Da Silva (1996), dans Eduardo Yazigi, Ana Fani Alessandri Carlos, Rita de Cassia A. Da Cruz (dir.), Turismo, sma pratica entre a crise e a inovaçao na metropole do Rio de Janeiro, Turismo, espaço, paisagem e cultura, Säo Paulo, Hucitec, p. 213-230.

Salomon, Jean-Noèl (1992), * Le complexe touristico-industriel d'Iguaçu-Itaipu (Argentine-Bresil-Uruguay) *, Bordeaux, Cahiers d'Outre-Mer, 45 (177), janvier-mars, p. 5-20.

Santos, Milton (1994), Por uma economia polisica da cidade : o caso de Sa Paulo, Hucitec, Săo Paulo, 145 p.

Santos, Milton (1992), \& Sa Paulo, un centre à la périphérie s, dans F. Durand, J. Levy et D. Retaille, Le Monde, Espaces et Systèmes, Paris, Dalloz.

Serson, Julio (2000), « Açao estratégica no turismo: o caso de São Paulo * dans Lage Cesar; H.G. Beatriz et Paulo C. Milone (dir), Turismo, teoria e pratica, São Paulo, Atlas, p. 233-239.

Serson, Julio (1993), * Meca para o turismo de negocios e eventos $\infty$, Turismo em analise, novembre, Escola de Comunicaçao e Artes. Universidade de São Paulo, p. 81-85.
Teixeira, I. (1994), a Os dolares que o Brasil perde com a violência $\%$, Conjuntura Econômica, Rio de Janeiro, septembre, p. 5961.

\section{Journaux et guides touristiques}

Brasilia Convention \& Visitors Bureau et Embratur, Perfil do turista do Distrito Federal (IBPC, 5 e enquête, ler trimestre 2001), 44 p. Embratur, Brasilia Convention \& Visitors Bureau, Imagens Editora, Brasilia, Tourist Guide $200 \mathrm{I}, 146 \mathrm{p}$.

Embratur, Instituto Brasileiro de Turismo, Ministério do Esporte e Turismo, Anuario Estatistico 2000, 208 p.

Embratur, Instituto Brasileiro de Turismo, Ministério do Esporte e Turismo, Estudo da demanda turistica international, (publication annuelle : dernière année consultée 1999).

Kern, Lara et Pimentel, Emani Figueiras (2000), Brasilia secreta, Portico, 112 p.

Organisation Mondiale du Tourisme (OMT) (1999), Tendencias del mercado turistico Américas 1989-1998. Madrid, OMT, $33^{\circ}$ réunion à Salvador de Bahia (Brésil), 24 juillet, graphiques et tableaux.

Rio Convention \& Visitors Bureau, dossier et site Internet.

Secretaria de Esportes e Turismo do Estado de Sĩo Paulo, Pesquisa sobre a Denanda Twristica da Cidade de São Paulo 2000, 22 tableaux.

Secretaria de Esportes e Turismo do Estado de São Paulo, Pesquisa sobre a Demanda Turística Internacional (enfoque Estado de São Paulo) - 1999, 17 tableaux.

\section{Quotidiens et hebdomadaires}

Carta do Rio, Rio de Janeiro.

Epoca.

Folha de São Paulo-FSP.

Jornal do Brasil - JB, Rio de Janeiro.

Gazeta Mercantil - GM, São Paulo.

O Estado de São Paulo-OESP, São Paulo.

O Globo, Rio de Janeiro.

Veja. 